\title{
Studien über Quercetin und seine Derivate
}

\author{
(VI. Abhandlung) \\ vou \\ Dr. J. Herzig.
}

Aus dem 1. Chemischen Universitätslaboratorium in Wien.

(Vorgelegt in der Sitzung am 8. Mai 1891.)

\section{Die Moleculargrösse des Quercetins.}

Durch eine sorgfältige Untersuchung der Alkyl- und Acetylalkylderivate des Quercetins habe ich seinerzeit ${ }^{1}$ gezeigt, dass der einfachste Ausdruck für die Moleculargrösse des Quercetins 292 ist. Daraus hat sich als Consequenz ergeben, dass von allen für das Quercetin vorgeschlagenen Formeln nur die von Löwe, $\mathrm{C}_{15} \mathrm{H}_{12} \mathrm{O}_{7}$, richtig sein kann, weil nur diese allein mit der experimentell ermittelten Moleculargrösse in Einklang gebracht werden kann. Die Frage nach der wirklichen Grösse des Quercetinmoleküls musste aber selbstverständlich offen bleiben und die Entscheidung darïber hing wesentlich von der Rolle ab, die man dem Sauerstoffatom zutheilte, welches sich im Quercetinmolekül wohl acetyliren, aber nicht alkyliren liess. In Übereinstimmung mit dem beim Chinon und seinen Derivaten bekannten Übergang in Hydrochinonderivate beim Acetyliren, nahm ich, ebenso wie beispielsweise Buchka beim Galler̈n, auch im Quercetin zwei Chinonsauerstoffe an, und demzufolge war es nothwendig, die Löw e'sche Formel zir verdoppeln. Das. Molekiul des Quercetins wäre dann 608, und es war sehr unwahr-

1 Monatshefte für Chemie, IX, 537 . 
scheinlich durch eine der neueren Methoden zur Bestimmung der Moleculargrộsse zu einem halbwegs brauchbaren Resultat zu gelangen. Aus diesem Grunde sind damals derartige experimentelle Bestimmungen unterblieben.

Diese Frage ist nun jetzt durch meine Erfahrungen beim Euxianthon in ein ganz anderes Stadium getreten. Es ist dadurch gezeigt worden, dass es durchaus nicht nothwendig ist, zur Erklärung dieses sonderbaren Verhaltens die Anwesenheit zweier Chinonsanerstoffe heranzuziehen. Eine Hydroxylgruppe verhält sich, wie wir gesehen haben, unter gewissen Verhältnissen bei der Allkylirung genau so, wie ich es seinerzeit beim Quercetin beschrieben habe. Berücksichtigt man fernerhin die sonstigen Analogien, die zwischen Quercetin und Euxanthon constatirt werden konnten, so kommt man zu dem Schlusse, dass in den Acetylalkylquercetinen höchst wahrscheinlich nur eine Acetoxylgruppe vorhanden sein wird, dass daher nach meinen Bestimmungen die Moleculargrösse des Quercetins 292 ist, und dass endlich consequenterweise die einfache, nicht verdoppelte Löw e'sche Formel die grösste Wahrscheinlichkeit für sich hat. Allerdings muss die alte Formel von Löwe etwas modificirt werden, da die analytischen Daten, sowie manche andere Reactionen mehr fiur $\mathrm{C}_{15} \mathrm{H}_{10} \mathrm{O}_{7}$ als wie für $\mathrm{C}_{15} \mathrm{H}_{12} \mathrm{O}_{7}$ sprechev.

Nun konnte man auch mit einiger Aussicht auf Erfolg daran denken, die Frage durch Moleculargewichtsbestimmungen lösen zu können, und ich bin Herrn Prof. Ostwald selrr dankbar, der zwei Bestimmungen nach Beckmann in seinem Laboratorium ausführen liess.

Herr stud. R. Bach, der die Versuche unternahm, theilt mir hieriiber Folgendes mit:

"Quercetin.

Lösungsmittel ...............Alkohol

Menge des Lösungsmittels .........35.50g

Angewandte Substanz........... $0.5606 \mathrm{~g}$

Siedepunktserhöhung .......... $0.07^{\circ}$

m. gefunden ..............258

m. berechnet . . . . . . . . . . . . . . 302. 
Acetyläthylquercetin.

Lösungsmittel ............... Äthylacetat

Menge des Auflösungsmittels . . . . . 39.68 $g$

Angewandte Substanz......... 1.1587 $g$

Siedepunktserhöhung ......... $0 \cdot 190^{\circ}$

m. gefunden . . . . . . . . 402 .

Hierauf stieg das Thermometer plötzlich, da der Barometerstand sich änderte. Nachdem das Thermometer wieder constant geworden war, erhielt ich auf Zusatz von $0.5757 \mathrm{~g}$ eine weitere Siedepunktserhöhung von $0.085^{\circ}$. Hieraus berechnet sich das Moleculargewicht zu 450. Die gesammte Erböhung von $0 \cdot 275^{\circ}$, hervorgerufen durch $1.7344 \mathrm{~g}$ Substanz, ergibt ein Moleculargewicht von 416 , berechnet $456 . "$

Durch diese Bestimmungen ist die Wahrscheinlichkeit für die Richtigkeit der Löwe'schen Formel um ein Bedeutendes erhöht worden. Trotzdem werde ich nicht ermangeln, dieselbe durch weitere Versuche nach anderen Methoden fester zn begrïnden.

Die folgende Zusammenstellung soll zeigen, in wie weit die bisher mit Sicherheit ermittelten analytischen Daten mit den für die Formel $\mathrm{C}_{15} \mathrm{H}_{10} \mathrm{O}_{7}$ geforderten tibereinstimmen.

Berechnet

1. Quercetin $\mathrm{C}_{15} \mathrm{H}_{10} \mathrm{O}_{7}$

2. Acetylquercetin

$\mathrm{C}_{15} \mathrm{H}_{5} \mathrm{O}_{2}\left(\mathrm{OC}_{2} \mathrm{H}_{3} \mathrm{O}\right)_{5}$

3. Methylquercetin

$$
\mathrm{C}_{15} \mathrm{H}_{6} \mathrm{O}_{3}\left(\mathrm{OCH}_{3}\right)
$$

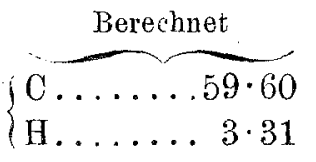

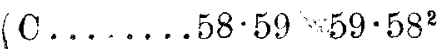$$
\text { H...... 3.90 } 3 \cdot 75
$$

Verseifung $58 \cdot 98 \quad 58 \cdot 73^{3}$

$\left\{\begin{array}{l}\mathrm{C} \ldots \ldots 63 \cdot 68 \\ \mathrm{H} \ldots \ldots .5 \cdot 03\end{array}\right.$

$63 \cdot 72^{2}$

$4 \cdot 93$

$62 \cdot 68^{2} \quad 62 \cdot 71^{5}$

$4 \cdot 90$

$89 \cdot 20^{4}$

$x$ Herzig, Monatshefte für Chemie, VI, S. 863.

2 Ebenda, V, S. 72.

3 Li ebermann, Berl. Berichte, XVII, S. 1680.

4 Herzig, Monatshefte für Chemie, IX, S. 537.

5 Ebenda, IX, S. 548. Aus Rhamnelin. 


$$
\begin{aligned}
& \text { Berechnet } \\
& \text { 5. Äthylquercetin } \quad \text { C ......66.66 } \\
& \mathrm{C}_{15} \mathrm{H}_{6} \mathrm{O}_{3}\left(\mathrm{OC}_{2} \mathrm{H}_{5}\right)_{4} \quad \text { H.....6. } 6.28 \\
& \text { Gefunden im } \\
& \text { Mittel } \\
& 66 \cdot 41^{1} \\
& 6 \cdot 23 \\
& \text { 6. Acetyläthylquercetin } \\
& \text { (C......65.79 } \\
& \mathrm{C}_{15} \mathrm{H}_{5} \mathrm{O}_{2}\left(\mathrm{OC}_{2} \mathrm{H}_{5}\right)_{4}\left(\mathrm{OC}_{2} \mathrm{H}_{3} \mathrm{O}\right) \quad \begin{array}{l}
\mathrm{H} \ldots \ldots .6 .14 \\
\text { Verseifung } 90 \cdot 78
\end{array} \\
& 6 \cdot 14 \\
& 90 \cdot 65
\end{aligned}
$$

Beim Bronderivat des Quercetins ergeben sich grossere Differenzen als zulässig sind. Die Maximaldifferenz ist $1.2 \% \mathrm{im}$ Bromgehalt. Dabei ist jedoch zu bemerken, dass dieser Körper und das Acetylderivat desselben sehr schwer rein darzustellen sind, so zwar, dass es weder Liebermann noch mir gelungen ist, bei den verschiedenen Darstellungen genügend constante Zahlen zu bekommen. Bei einem erneuerten Studium dieser Derivate wird übrigens auch diese Differenz sich möglicherweise beheben lassen.

Ich habe seinerzeit nachgewiesen, dass der Farbstoff der Gelbbeeren, Rhamnetin, ein Methylderivat des Quercetins ist, und zwar hielt ich es, gemäss meiner damaligen Anschauung ibber die Moleculargrösse des Quercetins für Dimethylquercetin. Nimmt man die Formel des Quercetins mit $\mathrm{C}_{15} \mathrm{H}_{10} \mathrm{O}_{7}$ als wahrscheinlich an, so ist das Rhamnetin ein Monomethylquercetin und die bereits publicirten Analysen stimmen mit dieser Annahme so ziemlich tiberein.

$$
\begin{aligned}
& \text { Berechnet } \\
& \text { C ....60.39 } \\
& \text { Rhamnetin } \\
& \mathrm{C}_{15} \mathrm{H}_{9} \mathrm{O}_{6}\left(\mathrm{OCH}_{3}\right)
\end{aligned}
$$

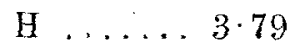

$$
\begin{aligned}
& \mathrm{OCH}_{3} \ldots 6 \cdot 40 \\
& \text { C ...... 59.50 } \\
& \text { H. . . . . . 4. } 43 \\
& \mathrm{O}_{15} \mathrm{H}_{5} \mathrm{O}_{2}\left(\mathrm{OCH}_{3}\right)\left(\mathrm{OC}_{2} \mathrm{H}_{3} \mathrm{O}\right)_{4}\left\{\begin{array}{l}
\mathrm{OCH}_{3} \ldots .9 \cdot 81 \\
\text { Verseifung 65 65 } 29
\end{array}\right. \\
& \text { Gefunden im } \\
& \text { Mittel } \\
& \overbrace{59 \cdot 92^{2}} \\
& 3 \cdot 94 \\
& 6 \cdot 47 \\
& 59 \cdot 00^{2} \\
& 4 \cdot 14 \\
& 9 \cdot 84 \\
& 65 \cdot 03
\end{aligned}
$$

1 Herzig, Monatshefte für Chemie, V, S. 72.

2 Ebenda, X, \$. 548. 
Was die Formel des Quercitrins betrifft, so entsteht die Frage, ob die Rhamnose darin in der Form $\mathrm{C}_{6} \mathrm{H}_{12} \mathrm{O}_{5}$ oder in der ihres Hydrats $\mathrm{C}_{6} \mathrm{H}_{14} \mathrm{O}_{6}$ rorhanden ist.

Die Entseheidung ist auf Grund der bisherigen Daten nicht möglich, da die Zahlen gerade in der Mitte liegen zwischen den theoretisch furr diese beiden Möglichkeiten geforderten. Durch ein erneuertes Studium des Quercitrins wird es sich aber wohl ermitteln lassen, da die von der Theorie verlangten Zahlen doch immerhin bis zu $1.5 \%$ von einander differiren.

Hiemit glaube ich, die Formel $\mathrm{C}_{15} \mathrm{H}_{10} \mathrm{O}_{3}$ für das Quercetin ziemlich wahrscheinlich gemacht zu haben, obwohl ich noch einzelner kleiner Lücken in der Beweisfuhrung mir wohl bewusst bin. Ich werde bestrebt sein, dieselben zu ergänzen und, wenn möglich, noch weitere Beweise dafür zu erbringen. Einen solchen Beweis möchte ich vorgreifend schon hier erwähnen. Es war bekannt, dass der Farbstoff des Fisetholzes, Fisetin, in naher Beziehung zum Quercetin steht, und J. Schmid ${ }^{1}$ hat gefunden, dass das Fisetin sich vom Quercetin durch einen Mindergehalt der Gruppe $\mathrm{CO}_{2}$ unterscheidet. Er ging dabei beim Quercetin von der alten Liebermann'schen Formel $\mathrm{C}_{24} \mathrm{H}_{16} \mathrm{O}_{11}$ aus und bemerkt ausdrücklich, dass die Reactionen des Fisetins mit der Formel durchaus nicht stimmen, und dass dieselben vielmehr dahin deuten, dass das Quercetin ein Dioxyfisetin sei. Nimmt man nun die Formel des Quercetins mit $\mathrm{C}_{15} \mathrm{H}_{10} \mathrm{O}_{\gamma}$ als richtig an, so verschwindet diese Differcnz zwischen Analysenzahlen nud Reactionen beim Fisetin vollkommen. In meiner nächsten $\mathrm{Ab}$ handlnng werde ich zeigen, dass sowohl die Zahlen von Schmid, als die von mir gefundenen ganz gut auf die Formel $\mathrm{C}_{15} \mathrm{H}_{10} \mathrm{O}_{6}$ für das Fisetin stimmen, und dass damit auch die Reactionen des Fisetin vollkommen in Einklang zu bringen sind, so dass also in der That das Quercetin ein Monoxyfisetin wäre. Dieser Umstand scheint mir aber anderseits ein weiterer Beweis fiur die Richtigkeit der Formel $\mathrm{C}_{15} \mathrm{H}_{10} \mathrm{O}_{7}$ für das Quercetin zu sein.

1 Berl. Berichte, XIX, S. 1742. 